\title{
Diffusion in linear porous media with periodic entropy barriers: A tube formed by contacting spheres
}

\author{
Marco-Vinicio Vazquez, ${ }^{1}$ Alexander M. Berezhkovskii, ${ }^{2}$ and Leonardo Dagdug ${ }^{1, a)}$ \\ ${ }^{1}$ Departamento de Fisica, Universidad Autonoma Metropolitana-Iztapalapa, Mexico, 09340 Distrito \\ Federal, Mexico \\ ${ }^{2}$ Mathematical and Statistical Computing Laboratory, Division for Computational Bioscience, Center for \\ Information Technology, National Institutes of Health, Bethesda, Maryland 20892, USA
}

(Received 23 April 2008; accepted 13 June 2008; published online 23 July 2008)

[DOI: $10.1063 / 1.2955447]$

In this paper we study diffusion in a tube formed by periodic contacting spherical cavities of radius $R$ (Fig. 1) over the entire range of the entropy barrier height. On times when the mean squared displacement of a diffusing particle is much greater than the tube period $l$, the particle motion can be characterized by an effective diffusion constant $D_{\text {eff }}$, which is smaller than the particle diffusion constant $D$ in space with no constrains. When the tube period increases, the radius $a$ of the circular aperture connecting neighboring cavities decreases, $a=\sqrt{R^{2}-\left(l^{2} / 4\right)}, 0<l<2 R$. As a result, the entropy barrier increases, and the ratio $D_{\text {eff }} / D$ gets smaller. One can find $D_{\text {eff }}$ analytically for high and low entropy barriers. For high entropy barriers $D_{\text {eff }}$ has been derived in Ref. 1 . Here we derive $D_{\text {eff }}$ for low entropy barriers. We also run Brownian dynamics simulations to find $D_{\text {eff }}$ as a function of the ratio $a / R$ and to compare the numerical results with those predicted by different analytical expressions. The major goal of our analysis is to establish the range of applicability of different approximate expressions for $D_{\text {eff }}$.

For high entropy barriers (small apertures, $a \ll R$ ) Berezhkovskii, Zitserman, and Shvartsman (BZS) derived the following expression for the effective diffusion constant:

$$
D_{\mathrm{eff}}^{\mathrm{BZS}}=\frac{6 D a}{\pi R} .
$$

In the opposite limiting case of low entropy barrier, i.e., when $(R-a) \ll R$, one can find $D_{\text {eff }}$ by approximately reducing the three-dimensional problem of diffusion in the tube of varying cross section to an effective one-dimensional problem of diffusion along the tube axis. Significant progress in understanding the reduction has been made in recent years. ${ }^{2-5}$ Directing the $x$-axis along the center line of the tube, one can write an approximate one-dimensional effective diffusion equation as

$$
\frac{\partial c(x, t)}{\partial t}=\frac{\partial}{\partial x}\left\{D(x) A(x) \frac{\partial}{\partial x}\left[\frac{c(x, t)}{A(x)}\right]\right\},
$$

where $D(x)$ is a position-dependent diffusion coefficient, $A(x)=\pi[r(x)]^{2}$ is the cross-section area of the tube of radius $r(x)$, and $c(x, t)$ is the effective one-dimensional concentration of the diffusing particles at given $x$, which is related to the three-dimensional concentration $C(x, y, z, t)$ by

$$
c(x, t)=\int_{A(x)} C(x, y, z, t) d y d z
$$

Equation (2) with position-independent diffusion coefficient, $D(x)=D$, is known as the Fick-Jacobs (FJ) equation. ${ }^{6}$ Zwanzig $(\mathrm{Zw})$ derived an expression for $D(x)$ assuming that the tube radius $r(x)$ is a slowly varying function, $\left|r^{\prime}(x)\right| \ll 1,{ }^{2}$
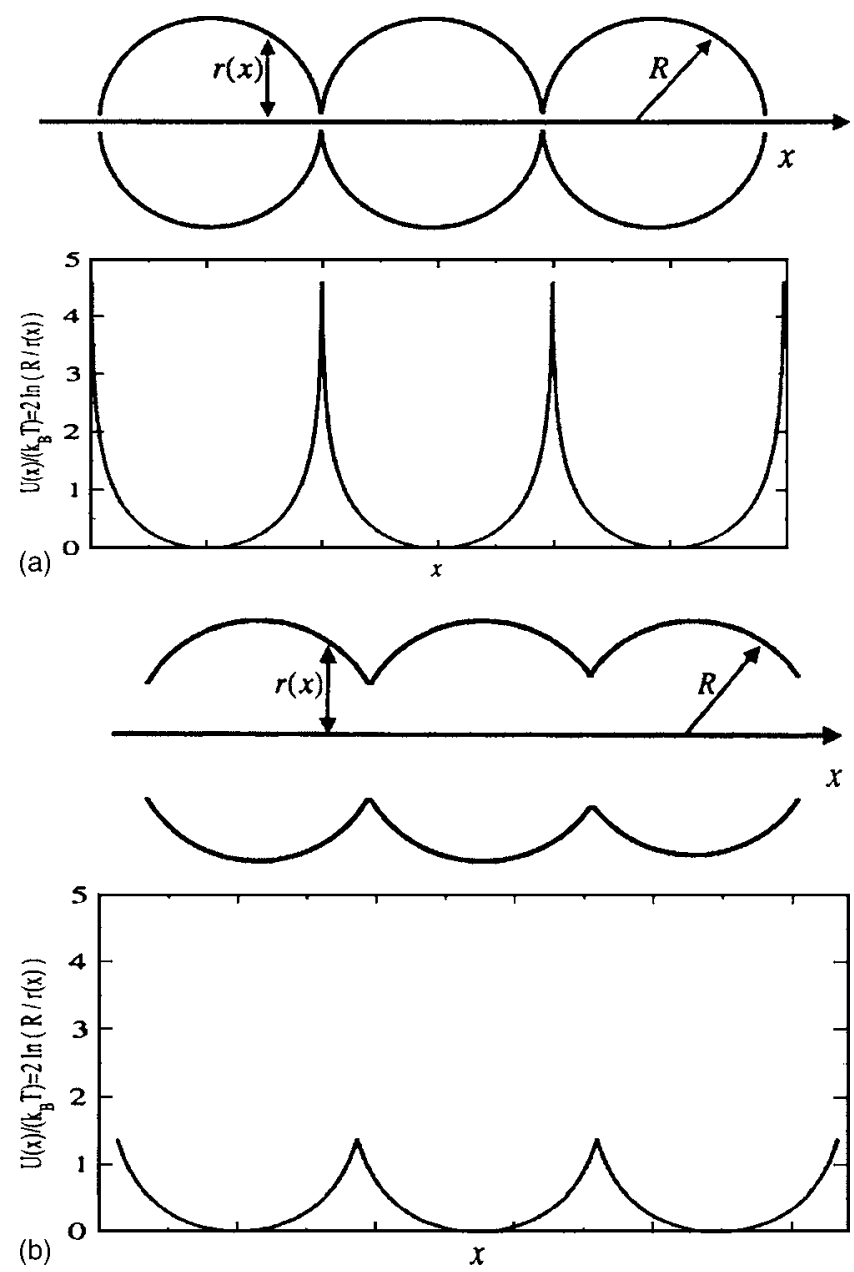

FIG. 1. Entropy potential for tubes with $(a / R)=0.1$ [panel (a)] and $(a / R)$ $=0.5$ [panel (b)]. The dimensionless heights of the entropy barriers are $\Delta U /\left(k_{B} T\right)=2 \ln 10=4.6[$ panel (a) $]$ and $\Delta U /\left(k_{B} T\right)=2 \ln 2=1.4$ [panel (b)]. 


$$
D_{\mathrm{Zw}}(x)=\frac{D}{1+r^{\prime}(x)^{2} / 2} .
$$

Reguera and Rubí (RR) generalized this result. ${ }^{3}$ Based on heuristic arguments they suggested

$$
D_{\mathrm{RR}}(x)=\frac{D}{\sqrt{1+r^{\prime}(x)^{2}}} .
$$

Equation (2) can be considered as the Smoluchowski equation for diffusion in the entropy potential $U(x)$ defined as

$$
U(x)=-k_{B} T \ln \frac{A(x)}{A\left(x_{0}\right)},
$$

where $k_{B}$ and $T$ are the Boltzmann constant and the absolute temperature, and $U(x)$ at $x=x_{0}$ is taken to be zero, $U\left(x_{0}\right)=0$. Potentials $U(x)$ with high and low entropy barriers are shown in Fig. 1. Since our system is periodic, it follows from Eqs. (4)-(6) that both $U(x)$ and $D(x)$ are periodic functions of $x$, $U(x+l)=U(x)$ and $D(x+l)=D(x)$. Therefore, we can find $D_{\text {eff }}$ using the Lifson-Jackson formula, ${ }^{7}$ which is an exact result for the one-dimensional Smoluchowski equation with periodic $U(x)$ and $D(x)$. According to this formula $D_{\text {eff }}$ is given by

$$
D_{\text {eff }}^{-1}=\left\langle[D(x) A(x)]^{-1}\right\rangle\langle A(x)\rangle,
$$

where $\langle f(x)\rangle=(1 / l) \int_{0}^{l} f(x) d x$. We use Eq. (7) to obtain three different expressions for $D_{\text {eff }}$.

Assuming that $D(x)=D$ we find $D_{\text {eff }}^{\mathrm{FJ}}$, which corresponds to the FJ equation

$$
\frac{D}{D_{\mathrm{eff}}^{\mathrm{FJ}}}=\left\langle\frac{1}{A(x)}\right\rangle\langle A(x)\rangle=\frac{2+(a / R)^{2}}{6 \sqrt{1-(a / R)^{2}}} \ln \frac{1+\sqrt{1-(a / R)^{2}}}{1-\sqrt{1-(a / R)^{2}}} .
$$

Using $D_{\mathrm{Zw}}(x)$ [Eq. (4)], we obtain $D_{\text {eff }}^{\mathrm{Zw}}$ given by

$$
\begin{aligned}
\frac{D}{D_{\mathrm{eff}}^{\mathrm{ZW}}}= & \frac{D}{D_{\mathrm{eff}}^{\mathrm{FJ}}}+\frac{1}{2}\left\langle\frac{r^{\prime}(x)^{2}}{A(x)}\right\rangle\langle A(x)\rangle \\
= & \frac{2+(a / R)^{2}}{12}\left[\frac{1}{(a / R)^{2}}\right. \\
& \left.+\frac{3}{2 \sqrt{1-(a / R)^{2}}} \ln \frac{1+\sqrt{1-(a / R)^{2}}}{1-\sqrt{1-(a / R)^{2}}}\right] .
\end{aligned}
$$

Respectively, $D_{\mathrm{RR}}(x)$ in Eq. (5) leads to $D_{\text {eff }}^{\mathrm{RR}}$ given by

$$
\frac{D}{D_{\mathrm{eff}}^{\mathrm{RR}}}=\left\langle\frac{\sqrt{1-r^{\prime}(x)^{2}}}{A(x)}\right\rangle\langle A(x)\rangle=\frac{2+(a / R)^{2}}{3(a / R)} .
$$

The results in Eqs. (8)-(10) were obtained assuming that the entropy barrier is low and the difference $R-a$ is small compared to $R$. It is interesting to compare the behavior predicted by these equations in the opposite limit when $a \rightarrow 0$ and the entropy barrier is high, with $D_{\text {eff }}^{\mathrm{BZS}}$ in Eq. (1), which is asymptotically exact in this limit. Comparison shows that $D_{\text {eff }}^{\mathrm{FJ}} / D_{\text {eff }}^{\mathrm{BZS}} \rightarrow \infty, D_{\text {eff }}^{\mathrm{ZW}} / D_{\text {eff }}^{\mathrm{BZS}} \rightarrow 0, D_{\text {eff }}^{\mathrm{RR}} / D_{\text {eff }}^{\mathrm{BZS}} \rightarrow \pi / 4$. Thus, $D_{\text {eff }}^{\mathrm{RR}}$ in Eq. (10) is a good candidate for a unique formula that

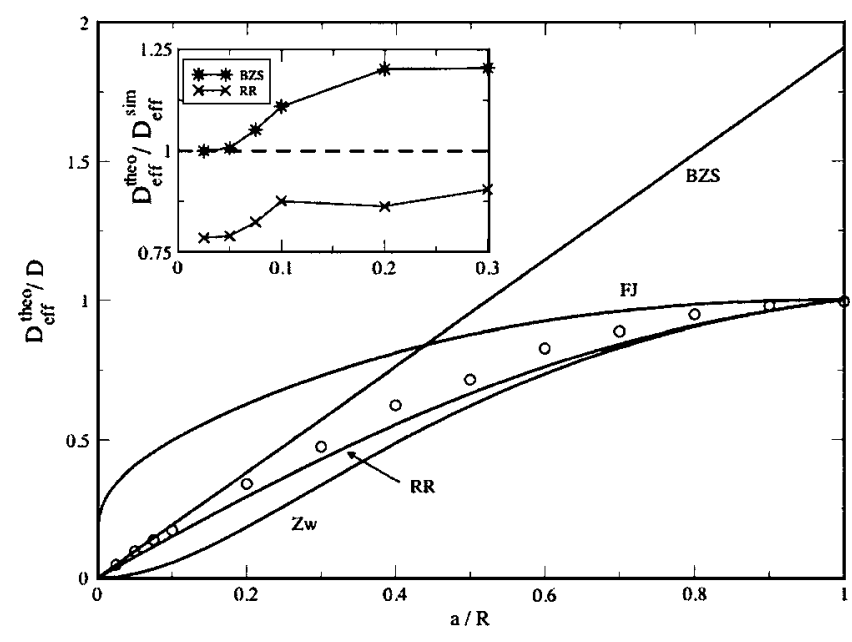

FIG. 2. Effective diffusion constants found numerically (circles) and predicted by Eqs. (1) and (8)-(10) (solid curves). The inset shows the ratio of $D_{\text {eff }}^{\text {BZS }}$ and $D_{\text {eff }}^{\text {RR }}$ predicted by Eqs. (1) and (10), respectively, to $D_{\text {eff }}^{\text {sim }}$ from $(a / R)=0.025$ to $(a / R)=0.3$.

covers the entire range of $a / R, 0<a / R<1$, while both $D_{\mathrm{eff}}^{\mathrm{FJ}}$ and $D_{\text {eff }}^{\mathrm{Zw}}$ fail as $a / R \rightarrow 0$.

We compare different expressions for $D_{\text {eff }}$ [Eqs. (1) and (8)-(10)], with $D_{\text {eff }}^{\text {sim }}$ found in Brownian dynamics simulations. Numerically we compute the mean squared displacement along the channel axis of $2.5 \times 10^{4}$ particles as a function of time, $\left\langle\Delta x^{2}(t)\right\rangle=\left\langle[x(t)-x(0)]^{2}\right\rangle$, assuming that the particle starting points are uniformly distributed over the cavity. We determine $D_{\text {eff }}^{\text {sim }}$ from the long-time behavior of $\left\langle\Delta x^{2}(t)\right\rangle$. The results presented in Fig. 2 show that $D_{\text {eff }}^{\text {sim }}$ is in excellent agreement with $D_{\text {eff }}^{\mathrm{BZS}}$ for $a / R<0.1$, reasonably well described by both $D_{\text {eff }}^{\mathrm{BZS}}$ and $D_{\text {eff }}^{\mathrm{RR}}$ for $a / R=0.2$, and close to $D_{\text {eff }}^{\mathrm{RR}}$ for $a / R \geqslant 0.3$.

To summarize, $D_{\text {eff }}^{\mathrm{RR}}$ in Eq. (10) found on the basis of the generalized Fick-Jacobs equation [Eq. (2)] with $D(x)$ given by the RR formula [Eq. (5)] provides a reasonably good approximation for $D_{\text {eff }}$ over the entire range of the size of the aperture. For small windows (high entropy barriers) $D_{\text {eff }}^{\text {sim }}$ found numerically is in excellent agreement with $D_{\mathrm{eff}}^{\mathrm{BZS}}$ in Eq. (1). We hope that the results of our analysis will be of use when interpreting experiments on controlled drug release and migration in porous media.

L.D. thanks CONACyT for partial support by Grant No. 52305. This study was partially supported by the Intramural Research program of the NIH, Center for Information Technology.

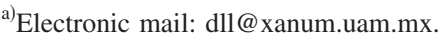

${ }^{1}$ A. M. Berezhkovskii, V. Yu. Zitserman, and S. Y. Shvartsman, J. Chem. Phys. 118, 7146 (2003); 119, 6991 (2003).

${ }^{2}$ R. Zwanzig, J. Phys. Chem. 96, 3926 (1992).

${ }^{3}$ D. Reguera and J. M. Rubí, Phys. Rev. E 64, 061106 (2001).

${ }^{4}$ P. Kalinay and J. K. Percus, J. Chem. Phys. 122, 204701 (2005); Phys. Rev. E 72, 061203 (2005); 74, 041203 (2006); J. Stat. Phys. 123, 1059 (2006).

${ }^{5}$ A. M. Berezhkovskii, M. A. Pustovoit, and S. M. Bezrukov, J. Chem. Phys. 126, 134706 (2007).

${ }^{6}$ M. H. Jacobs, Diffusion Processes (Springer, New York, 1967).

${ }^{7}$ S. Lifson and J. L. Jackson, J. Chem. Phys. 36, 2410 (1962).
} 